\title{
RAPID SYNTHESIS OF HIGHLY LUMINESCENT CARBON QUANTUM DOTS USING LOW-PRESSURIZED MICROWAVE SOLVOTHERMAL HEATING
}

\author{
K. Hagiwara and S. Horikoshi ${ }^{1}$
}

Department of Materials and Life Sciences, Faculty of Science and Technology, Sophia University, 7-1 Kioi-cho, Chiyoda-ku, Tokyo, Japan

k-hagiwara-7ge@eagle.sophia.ac.jp, 'horikosi@sophia.ac.jp

Keywords: Microwave Solvothermal Heating; Carbon quantum dots, Low-Pressurized synthesis

\section{Abstract}

In this research, we ameliorated the problems of carbon quantum dots synthesis and luminescence (quantum yields) by the novel synthesis protocol using microwave chemistry. Specifically, we synthesized high quantum yields carbon quantum dots $(61 \%)$ by utilizing a microwave chemical synthesis, synthesizing at low pressure condition (lower than 5 atom) and short reaction time $(3 \mathrm{hrs})$. The achievement of this high quantum yields made it clear that the contribution of polyethylene glycol (PEG) shell to carbon quantum dots is large. It was confirmed from the DLS and TEM image that the particle size of the synthesized particles was 8 to $13 \mathrm{~nm}$. On the other hand, the relationship between the polymerization degree of added PEG and the quantum yields to the addition amount is summarized. The quantum yields of carbon quantum dots without addition of PEG was $16.7 \%$, while it was improved at $61.1 \%$ when $0.6 \mathrm{~g}$ of PEG 6000 (Molecular weight: 6000) was added. Furthermore, we compared microwave with conventional method, and it was revealed that higher luminescent and narrower sized carbon quantum dots were synthesized in microwave heating than conventional method. This result presumably comes from the nature of microwave selective heating of carbon precursor.

\section{Introduction}

Since the first serendipity of carbon quantum dots (also called as carbogenic dots, carbon dots and carbon nanodots) ${ }^{1}$, it has been expected to be used for imaging materials for reusable living bodies ${ }^{2,3}$ (e.g. Hela cells) and detector for heavy metal ions ${ }^{4,5}$ (e.g. $\mathrm{Cu}^{2+}$, $\mathrm{Hg}^{2+}$ ), and many experimental protocols to synthesize carbon quantum dots have been reported, such as laser ablation ${ }^{6-8}$, electrochemical oxidation ${ }^{9-12}$, ultrasonic synthesis $^{13-16}$, and microwave heating ${ }^{17-20}$. However, in most of the reported methods, high temperature and pressure conditions and/or formation of solid-rich intermediates during the reaction are required to synthesize carbon quantum dots, which hinder the widespread of practical use of this material. Particularly, an acid-base reaction to synthesize carbon quantum dots using microwave heating leads the formation of solid-rich intermediates. For instance, succinic acid and tris (2-aminoethyl) amine are polymerized by microwave in the step of hydronation, resulting in the pyrolysis of black-colored polymer, followed by the purification using freeze-drying for 3 days ${ }^{20}$. This acid-base reaction also induces the long purification time and lowly luminescent products. To ameliorate these tasks, modified methodologies, such as solvothermal method using aromatic compounds ${ }^{21,22}$ and surface passivation using polymers ${ }^{5,6,23}$, have been reported recently.

Specifically, solvothermal method makes it possible to lower the production of toxic gas in the reaction system. For example, in 2018, L. Zhu and co-authors achieved in solvothermal-synthesizing carbon quantum dots with HATU/ other carbon sources and many kinds of low boiling point solvents, and in purifying the product using centrifugation ${ }^{21}$. 
This solvothermal method suppressed the production of solid-rich intermediates during the reaction and reduced purification time of the product. Also, surface passivation is known to bring higher quantum yields carbon quantum dots solution. For instance, Y. P. Sun and coworkers succeeded in functionalizing the surface of synthesized carbon quantum dots with PEG, resulting in the increase of quantum yields of carbon quantum dots ${ }^{6}$. Nonetheless, the existing carbon quantum dots synthetic methods have yet to be at the practical levels; the quantum yields are still lower than metal/semiconductor quantum dots, and synthetic condition is over $5 \mathrm{hrs}$ under more than $30 \mathrm{atms}^{24}$. Furthermore, carbon quantum dots synthesis using microwave solvothermal method, comparing to conventional solvothermal method, has not been reported yet, hence effects of microwave heating on the synthesis of carbon quantum dots have yet to be unveiled.

We successfully synthesized highly yellow luminescent carbon quantum dots (QYs: $61 \%$ at $580 \mathrm{~nm}$ ) by microwave solvothermal heating under much lower pressure condition (approximately 5 atm), using high boiling point solvent, ethylene glycol (b.p. $\left.197.6^{\circ} \mathrm{C}\right)^{25}$. This novel methodology also revealed the surface passivation of carbon quantum dots is highly dependent on the polymerization degree/amount of PEG. Furthermore, carbon precursor/ intermediate of carbon quantum dots highlighted a high absorbability of microwave, contributing to microwave superiority of carbon quantum dots synthesis over conventional solvothermal heating.

\section{Methodology}

In this research, highly luminescent carbon quantum dots were synthesized with 2step experimental strategies.

Synthesis of carbon precursor: White-colored solid anthracene $(0.143 \mathrm{~g}, 0.8 \mathrm{mmol})$ was added into $\mathrm{HNO}_{3}$ aq. $(20 \mathrm{~mL})$ in a three-necked round bottom flask $(50 \mathrm{~mL})$. The mixture was refluxed in $80{ }^{\circ} \mathrm{C}$ using microwave multimode chemical synthesis system (Wavemajic, EYELA; Frequency: $2.45 \mathrm{GHz}$ ). For comparison of microwave heating with conventional heating, oil bath heating was also conducted under the same conditions. In both microwave heating and conventional heating, samples were synthesized every hour for 12 hrs, and their changes over time were compared. Note that all the experiments were conducted in duplicates in the drying hood. After cooled to r.t., the mixture was centrifugated at $4000 \mathrm{rpm}$ for $5 \mathrm{~min}$. The resultant yellow 9.10-Dinitroanthracene was obtained by drying the precipitate at $60{ }^{\circ} \mathrm{C}$ with an electric furnace, followed by filtration with excess pure water, and Identification of the compound was performed by ${ }^{1} \mathrm{H}-\mathrm{NMR}$.

Synthesis of highly luminescent carbon quantum dots: 9.10-Dinitroanthracene $(0.107 \mathrm{~g}, 0.4 \mathrm{mmol})$ was added to $20 \mathrm{~mL}$ of ethylene glycol in a vial container. The mixture was turned into yellow solution after ultrasonicated $(20 \mathrm{kHz})$ for $5-10 \mathrm{~min}$ at room temperature. The yellow solution was removed and put into the reaction tube $(25 \mathrm{~mL}$ glass tube), then polyethlene glycol (PEG) and $\mathrm{NaOH}(0.24 \mathrm{~g})$ were added into the yellow solution. The polymerization degree of PEG used in this experiment was 600, 1500 and 6000 , respectively. The solution was rapidly heated to $210{ }^{\circ} \mathrm{C}$ using microwavesolvothermal method and kept at $210{ }^{\circ} \mathrm{C}$ for $3 \mathrm{hrs}$. In microwave solvothermal heating, single-mode applicator (Monowave 300, Anton Paar Ltd.; Frequency: $2.45 \mathrm{GHz}$ ) was utilized. Conventional solvothermal method was also carried out to for comparison with microwave solvothermal method. Impure carbon quantum dots solution (color: dark-blown) was obtained after rapidly cooling at r.t.. To remove impurities, the carbon quantum dots solution was centrifugated at $4000 \mathrm{rpm}$ for $5 \mathrm{~min}$ and dispersed in ethylene glycol. In order to investigate the solubility of the synthesized carbon quantum dots, carbon quantum dots synthesized without PEG were dissolved in four kinds of solvents; water, methanol, acetone and hexane. The ratio between carbon quantum dots solution and each solvent was fixed as 
1: 1 , and the surface status of carbon quantum dots was determined by FT-IR (FT/IR-4600, JASCO). The photoluminescence was evaluated by performing UV-Vis spectrometric measurement (V-7300, JASCO), 3D spectrum analysis (FP-8600, JASCO), and PL measurement (FP-8600, JASCO), and particle size distribution was characterized using TEM observation (H-7650, Hitachi High-Technologies; Accelerating voltage: $120 \mathrm{kV}$ )) and DLS analysis (Zetasizer nano ZS, Malvern). To determine microwave enhancement of carbon quantum dots $(0.60 \mathrm{~g}$ of PEG added $)$, temperature-dependent relative dielectric losses were measured with network analyzer (apparatus: E5071C, probe: 85070-20037, Keysight technology).

\section{Results and discussion}

Highly luminescent carbon quantum dots were synthesized by microwave solvothermal heating. In the determination of solubility of carbon quantum dots in four kinds of solvents (water, methanol, acetone and hexane), three ranges of emission colors (purple, blue and yellow) under UV irradiation were observed (Figure 1 (a)). On the ground that aromatic compounds emit from 370-440 nm (especially, anthracene strongly emits from $400-415 \mathrm{~nm})^{26}$, purple light was determined as the unreacted carbon precursor, 9.10Dinitroanthracene, or intermediates of carbon quantum dots. The other emission sources, on the other hand, were highly dissolved in water and water-soluble organic solvents (ethanol and acetone) but not in hexane, and were finally determined as carbon quantum dots. This observation implies that the surface of synthesized carbon quantum dots is polar and hydrophilic. It was also found that synthesized carbon quantum dots were completely dispersed into water, while, in the other water-soluble organic solvents, they were divided into two types of carbon quantum dots; emitting in the blue region and yellow emission region. For the further determination of the surface structure of synthesized carbon quantum dots, FT-IR measurement was utilized. According to Figure 1 (b), $-\mathrm{OH}$ and $-\mathrm{NO}_{2}$ moieties were observed at 2500-3600, 1520-1680, 1290-1360 $\mathrm{cm}^{-1}$ respectively, indicating that waterdispersibility of the synthesized carbon quantum dots derives from the surface structure of $\mathrm{OH}$ (hydroxy base), which is polar and hydrophilic. From these results, it was suggested that two types (blue and yellow emissions) of carbon quantum dots were both synthesized and characterized as water-dispersible due to the surface substituents of $-\mathrm{OH}$ moiety.
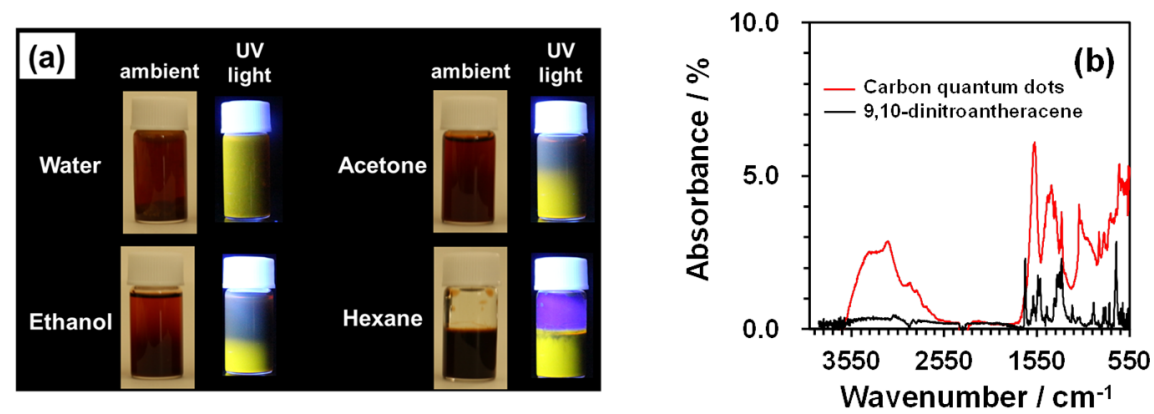

Figure 1. (a) solubility of carbon quantum dots to several kinds of solution: water, ethanol, acetone and hexane were each added to impure carbon quantum dots solution. The ratio between solution and a solvent was fixed as 1:1. (b) FT-IR spectra of non-PEG added carbon quantum dots solution. Each peak was assigned both automatically.

From the viewpoint of highly polarized surface of carbon quantum dots, purified carbon quantum dots were dispersed in water or ethylene glycol after centrifugation and used to measure the further analyses. From 3D-reverse, PL and UV-Vis spectroscopies, the 
optimum excitation wavelength for synthesized carbon quantum dots was determined as 275 $\mathrm{nm}$ by 3D-reverse analysis and PL spectra (Figure 2 (a)). Thus, the excitation wavelength of carbon quantum dots solution synthesized with various polymerization degree/ amount of PEG addition were also adjusted to $275 \mathrm{~nm}$ when PL spectra, full width at half maximum and quantum yields were measured. PL spectra was also utilized for the determination of the nature of carbon quantum dots. According to Table 1, a tendency of broader full width at half maximum was seen in all the experimental conditions, whistle quantum yields of each condition was of great difference. Quantum yields were improved dramatically as the added polymerization degree/ amount of PEG was changed, and we assume that this tendency derives from either the nature of microwave heating or high temperature condition. Moreover, it was observed that TEM image and DLS measurement show that the size distribution of synthesized carbon quantum dots is quite narrow (around 8-13 nm), indicating that a broad peak at approximately $580 \mathrm{~nm}$ depends on the nature of carbon source (e.g. surface emission), not on the widely distributed size of carbon quantum dots (Figure 2 (b)). This wide full width at half maximum can result from either large number of absorbance peaks or broadly distributed sizes of carbon quantum dots.
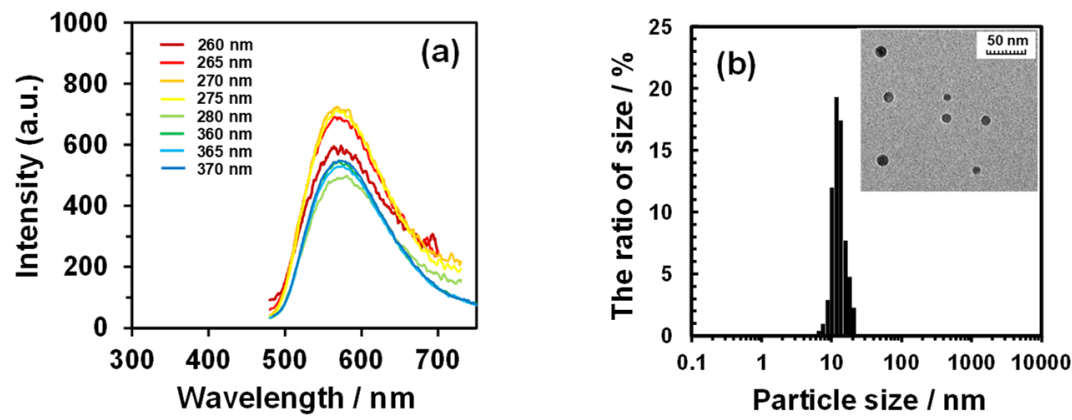

Figure 2. (a) PL spectrum and (b) TEM image of carbon quantum dots synthesized without the addition of PEG.

Table 1. Comparison between the amounts and kinds of polyethylene glycol (PEG) and the quantum yields (QYs) of synthesized carbon quantum dots solutions.

\begin{tabular}{cccc}
\hline Molecular weight of PEG & Weight amount /g & $\begin{array}{c}\text { FWHM / } \\
\mathrm{nm}\end{array}$ & QYs / \% at 275 nm \\
\hline$/ /$ & 0 & 108.8 & 16.7 \\
\hline 600 & 0.06 & 116.3 & 32.5 \\
\hline 600 & 0.15 & 115.5 & 42.9 \\
\hline 1500 & 0.15 & 114.4 & 39.9 \\
\hline 6000 & 0.15 & 114.8 & 43.1 \\
\hline 6000 & $\mathbf{0 . 6 0}$ & 112.2 & $\mathbf{6 1 . 1}$ \\
\hline
\end{tabular}

As aforementioned, high boiling point solvent, ethylene glycol, was utilized in this research. Note that this solvent plays an important role in lowering the inner pressure of reaction vessel, succeeding in the synthesis of highly luminescent carbon quantum dots and causing microwave-enhanced rapid heating of the solution. Particularly, ethylene glycol is well known as highly microwave absorbing solvent $\left(\varepsilon^{\prime \prime}=16.46\right.$ at $\left.25.0^{\circ} \mathrm{C}\right)$ and promoted the rapid heating of microwave: the heating rate of the solution was optimized as approximately 
$120{ }^{\circ} \mathrm{C} / \mathrm{min}$ in microwave heating and $1{ }^{\circ} \mathrm{C} / \mathrm{min}$ in conventional heating, resulting in the achievement of the 120 -fold faster heating rate. Thus, optimized total reaction time was set as $3 \mathrm{hrs}$ in microwave and $5 \mathrm{hrs}$ in autoclave, achieving 0.6-fold shorter total reaction time of carbon quantum dots in microwave solvothermal method. Moreover, it was confirmed that carbon quantum dots synthesized by two types of heating media, microwave and conventional, have apparent difference in qualities. For instance, we also synthesized carbon quantum dots with $0.60 \mathrm{~g}$ of PEG 6000 added in autoclave (conventional heating), and lower luminescence (QYs: $42.3 \%$ ) and broader size-distribution (diameter: $10 \mathrm{~nm}$ and 300-1000 $\mathrm{nm})$ were apparently observed in the conventional synthesis of carbon quantum dots in comparison with microwave-synthesized carbon quantum dots (QYs: 61.1 \%, diameter: 2-9 $\mathrm{nm}$ ) (Figure 3 (a)). We assume that this obvious difference was caused by microwave superiority, which is either rapid heating of microwave or specific microwave absorption of the other sources. By intentionally changing the heating rate in microwave method to 1 ${ }^{\circ} \mathrm{C} / \mathrm{min}$ (total reaction time: $5 \mathrm{hrs}$ ) as conventional method, it was clarified that slowly microwave-heated carbon quantum dots also attained approximately $61 \%$ of the quantum yields. This result highlights that the difference between microwave and conventional heating is related with specific microwave absorption of the other sources. Hence, in order to reveal the accuracy of hypothesized specific microwave superiority, the dependence of relative dielectric losses of ethylene glycol and the carbon precursor mixed solution on temperature was measured by network analyzer (Figure 3 (b)). It was revealed that the microwave absorbability of ethylene decreases as temperature increases, whistle that of the carbon precursor mixed solution stagnates even in the increase of temperature. This indicates that the carbon precursor and nuclei of carbon quantum dots should be the key factor to obtain highly luminescent carbon quantum dots, and microwave selective heating in the reaction temperature $\left(210^{\circ} \mathrm{C}\right)$ made a contribution to the highly heated carbon precursor/ nuclei of carbon quantum dots.
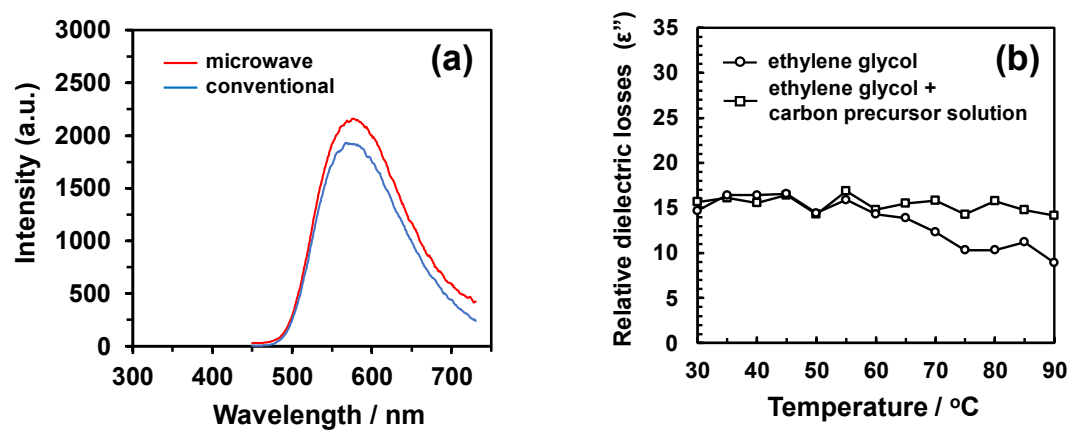

Figure 3. (a) Comparison of PL spectrum between microwave and conventional heating, and (b) comparison of relative dielectric losses between ethylene glycol and carbon quantum dots precursor solution.

\section{Concluding remarks}

We succeeded in achieving the uniform particle size distribution most required for nanomaterials, because the microwave method can rapidly heat the sample solution. This success made it clear that a rapid heating could result in high quantum yields. Particularly, the achievement of highly luminescent carbon quantum dots even by intentionally modified microwave slow heating clarified that relatively high microwave absorbability of carbon source in the reaction temperature is of great significance for microwave selective heating. 
Moreover, it was shown in this research that the mechanism of improvement of quantum yields differentiated from the polymerization degree/ amount of PEG.

\section{Acknowledgements}

We fully thank Prof. Kida of Kumamoto University for technical advice of this research. We also thank Prof. Uchida and Ass. Prof. Suzuki of Sophia University for the continuous support of analytical guidance. We also appreciate Prof. Torigoe of Tokyo University of Science for the TEM analysis.

\section{References}

1. X. Xu et al., J. Am. Chem. Soc., 2004, 126, 12736-12737.

2. H.M. Xiong et al., ACS Nano., 2016, 10, 484-491.

3. M. Zou et al., Mater. Lett., 201266 222-224.

4. B. Yang et al., Angew. Chemie - Int. Ed., 2013, 52, 3953-3957.

5. Chi Y et al., Anal. Chem., 2012, 84, 6220-6224.

6. Y. P. Sun et al., J. Am. Chem. Soc., 2006, 128, 7756-7757.

7. X. Wang et al., J. Phys. Chem. C., 2008, 112, 18295-18298.

8. N. Koshizaki et al., Chem. Commun., 2011, 47, 932-934.

9. K.P. Loh et al., ACS Nano., 2009, 3, 2367-2375.

10. Z. Ding et al., J. Am. Chem. Soc., 2007, 129, 744-745.

11. Z. Kang, Y. Liu, S.T. Lee et al., Angew. Chemie - Int. Ed., 2010, 49, 4430-4434.

12. Y. Liu, Z. Kang, S.T. Lee et al., ACS Appl. Mater. Interfaces, 2013, 5, 5080-5084.

13. Z. Kang et al, J. Mater. Chem., 2012, 22, 17470-17475.

14. J. Lee et al., ACS Appl. Mater. Interfaces, 2014, 6, 3365-3370.

15. Z. Kang et al, J. Chem., 36 (2012) 861-864.

16. S. Zhuo, M. Shao and S. T. Lee, ACS Nano, 2012, 6, 1059-1064.

17. W. Liu et al., J. Mater. Chem. B, 2014, 2, 46-48.

18. W. Guan, W. Gu et al., Int. J. Nanomedicine, 2014, 9, 5071-5078.

19. X. Sun, J. Mater. Chem., 2011, 21, 11726-11729.

20. I. In et al., RSC Adv., 2017, 7, 12663-12669.

21. L. Zhu et al, Chem. Sci., 2018, 9, 1323-1329.

22. X. G. Liu et al., Appl. Phys. Lett., 2015, 107, 213102-1-213102-5.

23. Y. Chen, Y. Yao et al., ACS Macro Lett., 2013, 2, 1033-1037.

24. X.B. Yin et al., Angew. Chemie., 2013, 19, 2276-2283.

25. K. Hagiwara, S. Horikoshi, 2019, will be published.

26. D. Jacewicz et al., Anal Lett., 2012, 46, 349-355. 